\title{
BD +36 3317: An Algol Type Eclipsing Binary in Delta Lyrae Cluster
}

\author{
O. Özdarcan*, E. Sipahi, H. A. Dal \\ Ege University, Science Faculty, Department of Astronomy and Space Sciences, 35100 \\ Bornova, İzmir, Turkey
}

\begin{abstract}
In this paper, we present standard Johnson $U B V$ photometry of the eclipsing binary BD +36 3317 which is known as a member of Delta Lyrae (Stephenson 1) cluster. We determined colors and brightness of the system, calculated $E(B-V)$ color excess. We discovered that the system shows total eclipse in secondary minimum. Using this advantage, we found that the primary component of the system has $B 9-A 0$ spectral type. Although there is no published orbital solution, we tried to estimate the physical properties of the system from simultaneous analysis of $U B V$ light curves with 2003 version of Wilson - Devinney code. Then we considered photometric solution results together with evolutionary models and estimated the masses of the components as $M_{1}=2.5 M_{\odot}$ and $M_{2}=1.6 M_{\odot}$. Those estimations gave the distance of the system as $353 \mathrm{pc}$. Considering the uncertainties in distance estimation, resulting distance is in agreement with the distance of Delta Lyrae cluster. Keywords:

techniques: photometric - (stars:) binaries: eclipsing - stars: individual:
\end{abstract}

\footnotetext{
${ }^{*}$ Corresponding author

Email address: orkun.ozdarcan@ege.edu.tr (O. Özdarcan)
} 
$(\mathrm{BD}+363317)$

\section{Introduction}

$\mathrm{BD}+363317\left(\mathrm{SAO} 67556, \alpha_{2000}=18^{h} 54^{m} 22^{s}, \delta_{2000}=36^{\circ} 51^{\prime} 07^{\prime \prime}, V=\right.$ $\left.8^{m} .77\right)$ is an Algol type eclipsing binary which is in the same area with Delta Lyrae cluster $\left(\alpha_{2000}=18^{h} 54^{m}, \delta_{2000}=36^{\circ} 49^{\prime}\right.$, Kharchenko et al. (2005)) in the sky. The system is considered as a member of the cluster (Eggen, 1972, 1983; Anthony-Twarog, 1984). The existence of the cluster was suggested by Stephenson (1959) for the first time. Further photometric evidence for the existence of the cluster was provided by Eggen (1968). However, possible members of the cluster, included BD +36 3317, have considerably different distance modulus values (Anthony-Twarog, 1984) and this makes harder to confirm real members, even the existence of cluster. After all, mean distance modulus for the cluster is given as $7^{m} .29$ with $E(B-V)=0^{m} .04$ by Anthonv-Twarog (1984). More recent distance modulus value was given by Kharchenko et al. (2005) as $7^{m} .98$ (their $E(B-V)$ value is $0^{m} .04$, too) with the distance of 373 pc. Kharchenko et al. (2004) calculated the Delta Lyrae membership probability of $\mathrm{BD}+363317$ as $0.67,0.91$ and 1.0, in terms of proper motion, photometry and spatial position, respectively. This calculation seems to support the membership of BD +36 3317 to the cluster.

Spectroscopic binary nature of the star has been first noticed by Eggen (1968) via its large radial velocity variation from $-90 \mathrm{~km} \mathrm{~s}^{-1}$ to $17 \mathrm{~km} \mathrm{~s}^{-1}$. However, eclipses of the system has been discovered by Violat-Bordonau and Arranz Heras (2008), many years after the discovery of its spectroscopic binary nature. No orbital solution via radial velocity measurements has been published up to 
know. At that point, $\mathrm{BD}+363317$ has the advantage of to be an eclipsing binary in terms of estimating its absolute physical properties and distance. Furthermore, one can calculate its distance and compare it with the distance of the cluster and check the membership of the system to the cluster. For those purposes, we obtained standard Johnson $U B V$ observations of the system in 2008 and 2009. By using standard photometric data, we calculated $E(B-V)$ value of the system and estimated their spectral type. Although the lack of spectroscopic mass ratio and orbital solution is a disadvantage in terms of determination of the absolute dimension of the system, we made a fair estimation for physical properties of $\mathrm{BD}+363317$, including the distance of the system, by using the photometric solution. In the next section, we give summary of our observations. In section 3 we refine the light elements of the system via $O-C$ analysis. In section 4 , we give basic photometric properties of the system and investigate the effect of interstellar extinction. In section 5 we present the simultaneous analysis of $U B V$ light curves and our estimation for the absolute dimension of the system. In the last section we discuss the results.

\section{Observations}

We carried out Johnson $U B V$ observations of the star at Ege University Observatory $(E U O)$. We observed the star on 41 separate nights in 2008 and 2009. Our instrumental setup was $0.3 \mathrm{~m}$ Schmidth-Cassegrain telescope equipped with uncooled SSP5 photometer. BD +36 3314 was comparison star in our observations, while $\mathrm{BD}+363313$ was check star. In many observing run, when no primary or secondary minimum occurred, we only performed 
a short observing sequence as $S-C 2-C 1-V V V V-S-C 2-C 1-$ $V V V V-C 1-C 2-S$, where $S$ is sky, $C 2$ is check star, $C 1$ is comparison star and $V$ is variable star. For those kind of nights, we used average atmospheric extinction coefficients of EUO in order to correct all differential magnitudes in terms of $V-C 1, V-C 2$ and $C 2-C 1$. For other nights, when a primary or a secondary minimum occurred, we performed an all night observing run. For those nights, we calculate atmospheric extinction coefficients via measurements of $C 1$ and made all corrections for atmospheric extinction on differential magnitudes according to those coefficients. We estimate average standard deviations of observations from $C 2-C 1$ measurements and resulting average standard deviations are $0^{m} .053,0^{m} .019$ and $0^{m} .017$ for $U$, $B$ and $V$ filters, respectively. We observed 11 stars from IC 4665 cluster (Menzies and Marang, 1996) on $29^{\text {th }}$ July 2008 and 9 stars from the list of Andruk et al. (1995) on $17^{\text {th }}$ August 2009, in order to calculate coefficients of the transformation of the instrumental system to the standard one. By those coefficients, we applied color corrections for all differential measurements. Then, we directly calculated average standard magnitudes and colors of the comparison star from those two nights. Finally, by using standard magnitude and colors of the comparison star, we calculate standard magnitude and colors of the variable.

\section{3. $O-C$ Analysis}

It is not possible to make a comprehensive $O-C$ analysis for $\mathrm{BD}+36$ 3317 since there is not enough minimum time observations. Only the first ephemeris of the system is available in literature (Violat-Bordonau and Arranz Heras, 
2008) as

$$
(H J D)_{M i n I}=2,454,437.25921+4^{d} .30216 \times E .
$$

where $(H J D)_{M i n I}$ is epoch, which corresponds to a time of a primary minimum of $\mathrm{BD}+363317$, and $E$ is integer eclipse cycle number. We have already had three primary minima (Type I) and one secondary minimum (Type II) in our observations (Sipahi et al., 2009). In Table 1, we list those minima with corresponding $O-C$ values.

Table 1: The times of light minima of $\mathrm{BD}+36$ 3317. In the first column, the errors are given for the last digit of the measurements.

\begin{tabular}{|c|c|r|c|c|}
\hline $\begin{array}{c}\text { HJD } \\
(2400000+)\end{array}$ & E & $\begin{array}{r}O-C \\
(\text { day })\end{array}$ & Filter & Type \\
\hline $54652.3522(4)$ & 50.0 & -0.0025 & $U B V$ & I \\
$54667.4148(5)$ & 53.5 & 0.0026 & $U B V$ & II \\
$55052.4561(2)$ & 143.0 & 0.0004 & $U B V$ & I \\
$55078.2683(4)$ & 149.0 & -0.0004 & $U B V$ & I \\
\hline
\end{tabular}

Application of linear least squares method to primary and secondary minima data gives very small amount of corrections in the ephemeris. The new light elements and their errors are as follows:

$$
(H J D)_{M i n I}=2,454,437.2466(30)+4^{d} .302162(27) \times E .
$$

For further analysis of light curves, we use those final light elements. 


\section{Basic Photometric Parameters and Interstellar Extinction}

We show phased light and color curves of the system in Figure 1.

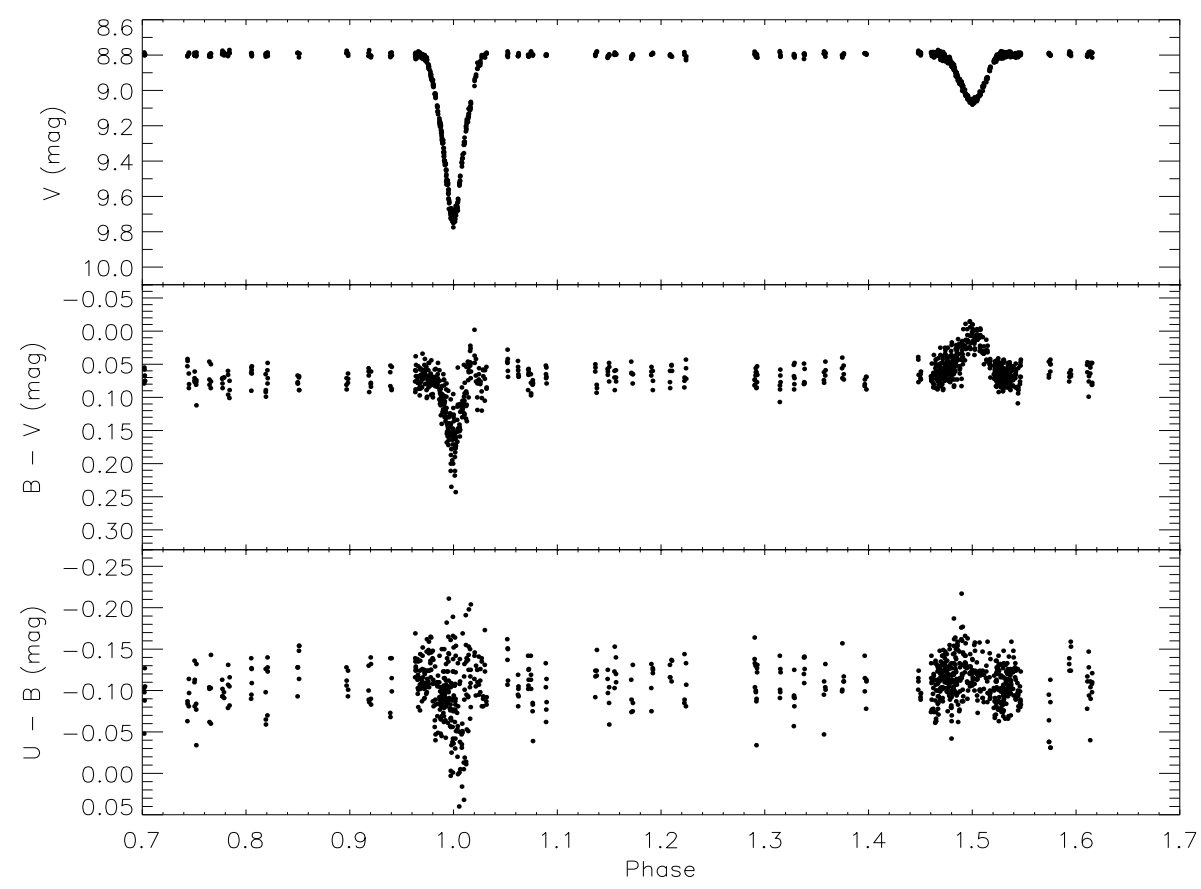

Figure 1: Phase folded and standardized light and color curves of BD +363317 .

One can easily notice clear variations in $B-V$ in primary and secondary minima, which indicate quite large temperature difference among the components of the system. We determined brightnesses and colors of the system in maximum light, primary and secondary minima, which would give us some hints about components. We list those values in Table 2 .

We can inspect effect of interstellar extinction via $U B V$ color - color diagram, by considering the colors at maximum light. We used $U B V$ standard star data from Drilling and Landolt (2000) for this purpose. First, we used 
Table 2: Magnitude and colors of BD +36 3317 .

\begin{tabular}{|c|c|c|c|}
\hline & $\begin{array}{c}\text { Max } \\
(\mathrm{mag})\end{array}$ & $\begin{array}{c}\text { Min I } \\
(\mathrm{mag})\end{array}$ & $\begin{array}{c}\text { Min II } \\
(\mathrm{mag})\end{array}$ \\
\hline $\mathrm{V}$ & 8.798 & 9.715 & 9.053 \\
U-B & -0.108 & -0.090 & -0.119 \\
$\mathrm{~B}-\mathrm{V}$ & 0.066 & 0.167 & 0.009 \\
\hline
\end{tabular}

magnitude and colors of maximum light (see Max column in Table 2) by assuming $E(U-B) / E(B-V)=0.72$ as the slope of the reddening vector in $U B V$ color - color diagram. Resulting $E(B-V)$ and $A_{v}$ values are $0^{m} .139$ and $0^{m} .43$, respectively, under the assumption of $R=3.1$. Then, de-reddened total color for the system is $(B-V)_{0}=-0^{m} .07$. Now we can make an estimation for the temperature of the primary component, by assuming that the maximum contribution to the total light comes from the primary component. However, during the light curve analysis, we noticed that orbital inclination value of the system shows very small variations around 89.4 degrees which makes the secondary minimum total eclipse. Then, the secondary component is completely hidden behind the primary component at the middle of the secondary minimum, hence, the magnitude and colors of that phase corresponds to the direct measurements of the primary component. Here, we refer the reader to the next section for justification of "total eclipse at secondary minimum" case. In this case, we have direct measurements of the primary component (third column in Table 2) and total colors and magnitude of the system (first column in Table 2), therefore we 
can easily calculate magnitude and colors of the secondary component as $V$ $=10^{m} .497, U-B=-0^{m} .053$ and $B-V=0^{m} .316$. This case is also an advantage in terms of more accurate determination of the interstellar reddening and intrinsic colors of the components separately. Therefore, we repeated the calculation of the interstellar reddening as described above, but this time by using the direct measurements of the primary component at the middle of the secondary minimum. We list intrinsic colors of the components together with the more accurate interstellar reddening and extinction estimation in Table 3 .

Table 3: Intrinsic colors and magnitude of the components of BD +36 3317 together with the amount of interstellar reddening and extinction.

\begin{tabular}{|c|c|c|}
\hline & $\begin{array}{c}\text { Primary } \\
(\mathrm{mag})\end{array}$ & $\begin{array}{c}\text { Secondary } \\
(\mathrm{mag})\end{array}$ \\
\hline$V_{0}$ & 8.833 & 10.277 \\
$(U-B)_{0}$ & -0.170 & -0.104 \\
$(B-V)_{0}$ & -0.062 & 0.245 \\
\hline$E(B-V)$ & \multicolumn{2}{|c|}{0.07} \\
$A_{v}$ & \multicolumn{2}{|c|}{0.22} \\
\hline
\end{tabular}

According to Table 3 , colors of the primary component indicates $B 9$ spectral type while secondary components corresponds about $A 8$ spectral type. 


\section{Analysis of Light Curves}

Under the assumption of total eclipse in secondary minimum, we can estimate the effective temperature of the primary component directly, which is very critical for light curve analysis. We adopted calibration of Gray (2005) for effective temperature estimation of the primary component and resulting temperature for $(B-V)_{0}=-0^{m} .062$ is $T_{1}=10750 \mathrm{~K}$ with the error of $\sigma_{T_{1}}=470 \mathrm{~K}$. The error of the temperature is calculated from the standard deviation of $B-V$ color in our observations.

Before starting analysis, we chose 0.25 phase as normalisation phase and converted all magnitude measurements into normalised flux according to the light level at that phase. For light curve analysis, we used 2003 version of the Wilson - Devinney code (Wilson and Devinney, 1971; Wilson, 1979, 1990). Photometric properties of the components gives us hints for some parameters to reduce the number of free parameters in photometric solution. We adopt the gravity brightening coefficients $g_{1}=g_{2}=1$ and albedos $A_{1}=A_{2}=1$ for stars which have radiative envelopes. We assume synchronised rotation for both components, so $F_{1}=F_{2}=1$. We took the band-pass dependent $\left(x_{1,2}, y_{1,2}\right)$ and bolometric $\left(x_{1,2}(b o l), y_{1,2}(b o l)\right)$ limb darkening coefficients from van Hamme (1993) by assuming square root law (Díaz-Cordovés and Giménez, 1992) which is more appropriate for stars hotter than $8500 K$. Since there is no radial velocity study in literature, we do not have any information about mass ratio, which is another critical parameter for light curve analysis. Although it is not an efficient way to determine $q$ from photometry in detached systems, we searched for the best solution for different $q$ values, starting from $q=0.30$ until $q=0.90$, by using $U B V$ 
data simultaneously. Orbital inclination $(i)$, effective temperature of the secondary component $\left(T_{2}\right), \Omega$ potentials of primary and secondary $\left(\Omega_{1}, \Omega_{2}\right)$ and luminosity of the primary component $\left(L_{1}\right)$ are free parameters in the solution. The errors of the best solutions for individual $q$ values are very close to each other between $q=0.45$ and $q=0.70$. At that point, we considered mass - luminosity relation as $L \propto M^{4}$ by using the resulting absolute luminosities at the end of the solution (see later results in this section). We repeated solutions for many $q$ values between $q=0.45$ and $q=0.70$. In most cases, $L \propto M^{4}$ relation indicates the $q$ value close to 0.65 , hence, we finally accepted $q=0.65$ and applied a final light curve solution. In Table 4, we give final light curve analysis results. We note that the error of $T_{2}$ is internal to the Wilson - Devinney code and its error should be similar to the error of $T_{2}$. In the table, $\left\langle r_{1,2}\right\rangle$ denotes average of three fractional radius values (pole, side and back values in solution output) relative to the semi-major axis of the orbit, for corresponding component. We give the normalised fluxes for $U, B$, and $V$ filters and corresponding theoretical light curves for our final solution in Figure 2,

In Figure 3, we zoom to the primary (left panels) and secondary (right panels) minima to show the shapes of the eclipses. One can notice that the secondary minimum is certainly total eclipse which lasts for a short phase range. At the primary minimum, we observe non-flat bottomed light variation which shows the effects of annular eclipse and limb darkening together.

Comparison among photometric solution results, mass - luminosity relation and evolutionary models of Girardi et al. (2000) indicates that the mass of the primary $M_{1}$ is close to the $2.5 M_{\odot}$, therefore we assume $M_{1}=2.5 M_{\odot}$. 
Table 4: Light curve analysis results of $\mathrm{BD}+36$ 3317. Errors are given in parenthesis.

\begin{tabular}{|c|c|}
\hline$q\left(=M_{2} / M_{1}\right)$ & 0.65 (fixed) \\
$T_{1}(K)$ & 10750 (fixed) \\
$g_{1}=g_{2}$ & 1.0 \\
$A_{1}=A_{2}$ & 1.0 \\
$F_{1}=F_{2}$ & 1.0 \\
$i\left(^{\circ}\right)$ & $89.61(11)$ \\
$T_{2}(K)$ & $7711(10)$ \\
$\Omega_{1}$ & $10.571(25)$ \\
$\Omega_{2}$ & $8.997(23)$ \\
$L_{1} /\left(L_{1}+L_{2}\right)_{U}$ & $0.851(17)$ \\
$L_{1} /\left(L_{1}+L_{2}\right)_{B}$ & $0.831(15)$ \\
$L_{1} /\left(L_{1}+L_{2}\right)_{V}$ & $0.789(15)$ \\
$x_{1}(b o l), x_{2}(b o l)$ & $0.558,0.215$ \\
$y_{1}(b o l), y_{2}(b o l)$ & $0.172,0.525$ \\
$x_{1}, y_{1}(U)$ & $0.082,0.590$ \\
$x_{1}, y_{1}(B)$ & $-0.058,0.846$ \\
$x_{1}, y_{1}(V)$ & $-0.047,0.723$ \\
$x_{2}, y_{2}(U)$ & $0.184,0.646$ \\
$x_{2}, y_{2}(B)$ & $0.105,0.822$ \\
$x_{2}, y_{2}(V)$ & $0.096,0.711$ \\
$\left\langle r_{1}\right\rangle,\left\langle r_{2}\right\rangle$ & $0.1009(3), 0.0832(2)$ \\
$\mathrm{rm}$ & 0.011 \\
\hline
\end{tabular}




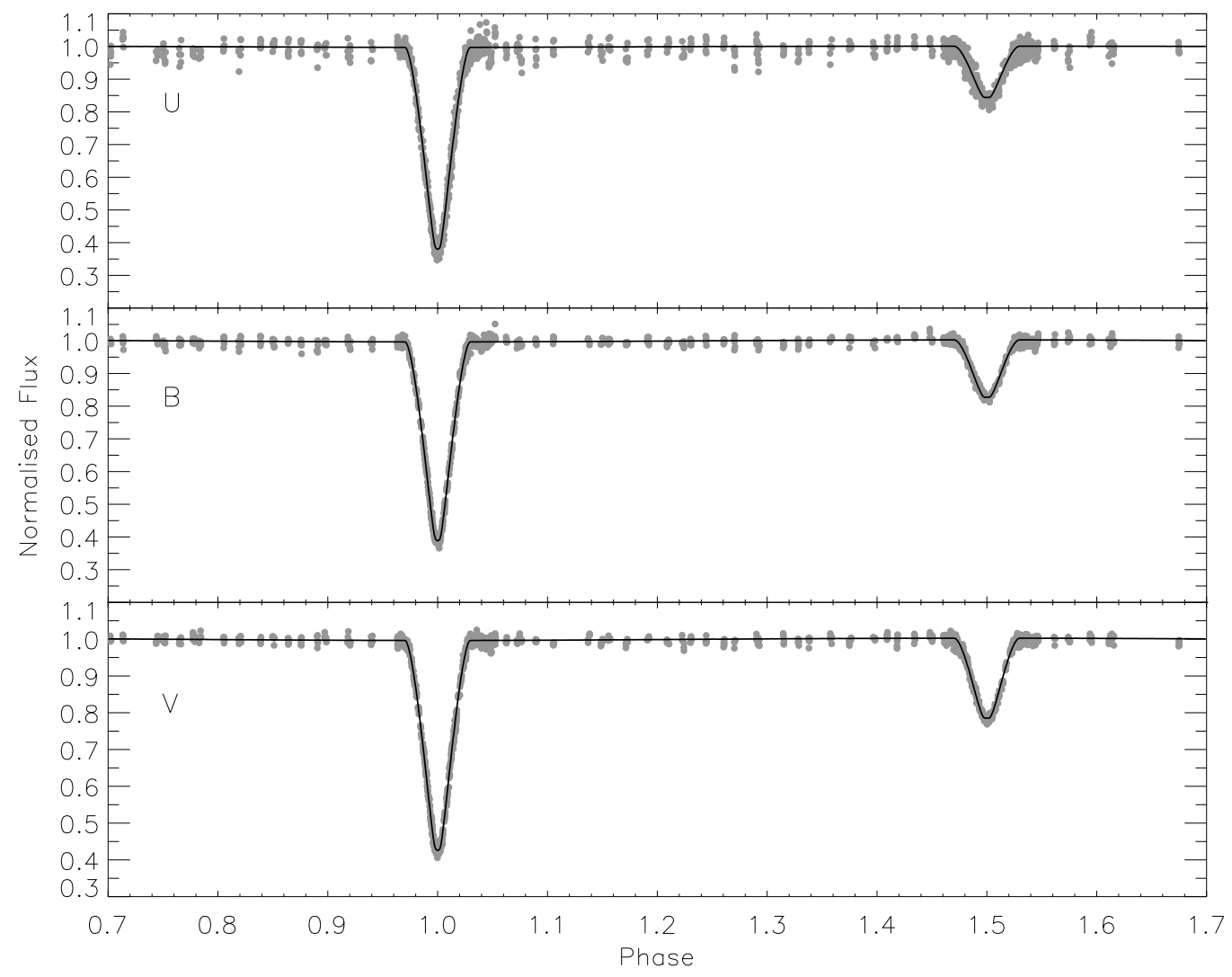

Figure 2: Representation of observational data (points) in terms of normalised flux and theoretical solution (continuous line).

This assumption makes the mass of the secondary component $M_{2}=1.6 M_{\odot}$ according to the $q$ value.

Since we estimated the masses of the components, we can go one step further and calculate the absolute parameters and the distance of the system. By the aid of Kepler's third law, we can calculate semi-major axis of the orbit $a$. After that point, one can easily calculate absolute radii of the components by using average fractional radii in Table 4. Now we have effective temperatures and absolute radii of the components and we can calculate 

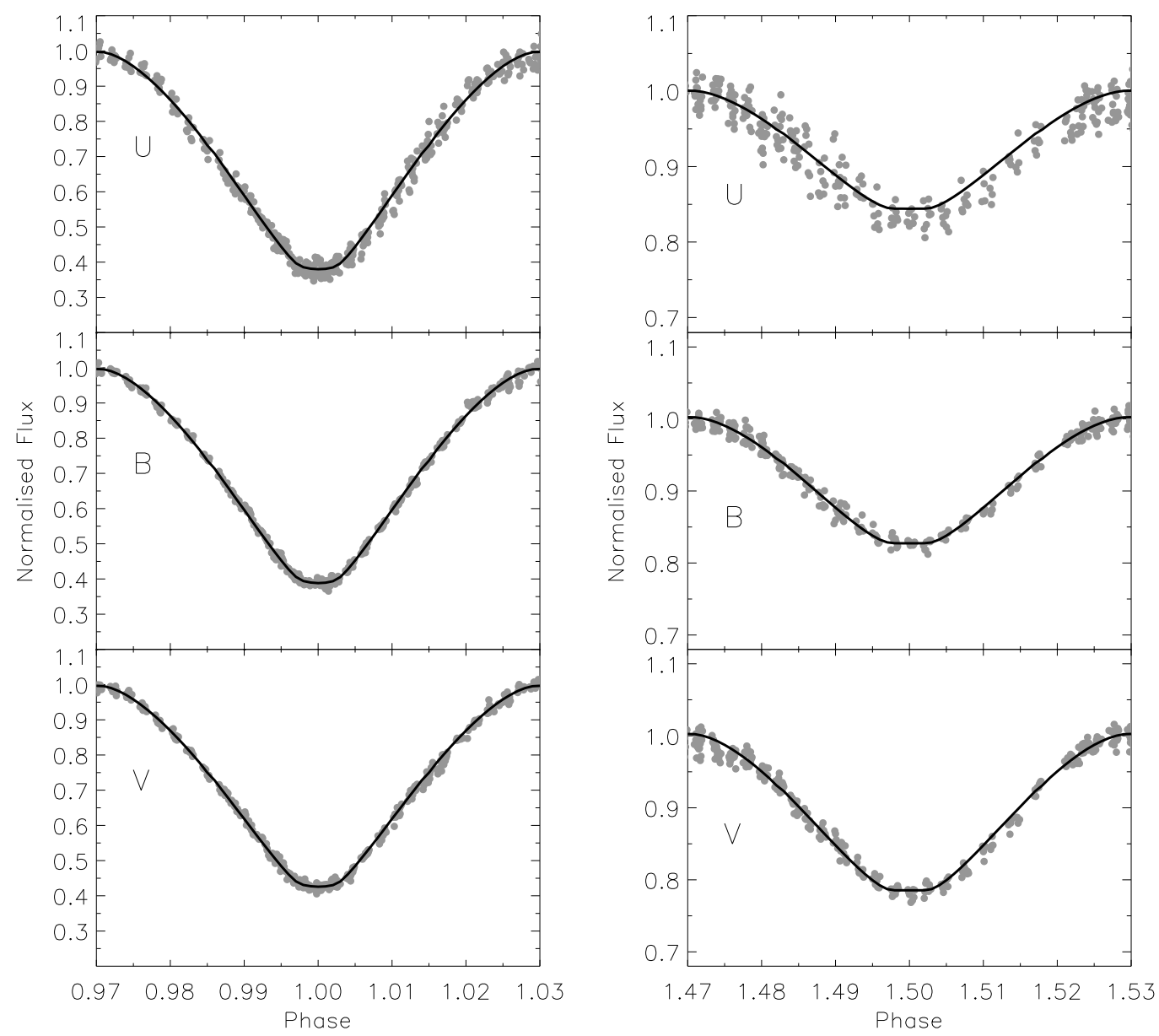

Figure 3: Close look to the primary and secondary minima in each filter. In secondary minimum, one can notice the short total eclipse.

their luminosities in solar unit via Stefan - Boltzmann law, by using $T_{\odot}=$ $5770 \mathrm{~K}$. We list absolute parameters of the system in Table 5 . Finally, we can calculate the distance of the system by using primary and secondary component separately, via their photometric and absolute properties. In distance calculation, we adopted bolometric corrections from Grav (2005). Photometric properties of the primary component leads to a distance of $353 \mathrm{pc}$. We 
only use primary component in order to estimate the distance since its signal is very strong relative to the secondary component.

Table 5: Absolute Physical Properties of BD +36 3317. Solar $M_{b o l}$ value of $4 .^{m} 74$ is used to calculate $M_{b o l}$ values of the components.

\begin{tabular}{|c|c|c|}
\hline Parameter & Primary & Secondary \\
\hline Mass $\left(M_{\odot}\right)$ & 2.5 & 1.6 \\
Radius $\left(R_{\odot}\right)$ & 1.8 & 1.5 \\
Luminosity $\left(L_{\odot}\right)$ & 39 & 7 \\
$M_{\text {bol }}(\mathrm{mag})$ & 0.76 & 2.62 \\
$\log (g)(\mathrm{cgs})$ & 4.32 & 4.31 \\
\hline
\end{tabular}

\section{Summary and conclusions}

We presented standard Johnson $U B V$ photometry of the Algol type eclipsing binary $\mathrm{BD}+363317$ with a fairly good phase coverage and reasonably accurate observational data. During observations, we obtained three primary and one secondary minima. We refined the epoch and the period of the system by applying linear least squares method to the timings of those light minima. We determined standard colors and magnitude of the system in maximum light, primary minimum and secondary minimum. During the analysis, we noticed that the secondary minimum is total eclipse, which is an advantage in analysis and means that the measurement at that phase corresponds to direct measurements of the primary component. Using this advantage, we first determined interstellar reddening and extinction via direct 
measurements of the primary component. Then, we were able to calculate de-reddened colors and magnitudes of the components, separately. This case was an another advantage in order to make a more accurate estimation of $T_{1}$, hence enabled us to reduce the number of free parameters more reliably in simultaneous $U B V$ light curve solution. Photometric solution justified that the secondary minimum was total eclipse.

Lack of an orbital solution based on radial velocity measurements prevented us from determining the accurate absolute parameters of the system and their uncertainties. Hence, we can only make a rough estimation for the uncertainties and check how those uncertainties affect our results.

If we assume that the $q$ is between 0.6 and 0.7 and $M_{1}$ is between $2.4 M_{\odot}$ and 2.6 $M_{\odot}$, then we can calculate a lower and upper limit for $a$ via Kepler's third law. Those lower and upper limits of $a$ put $R_{1}$ between $1.76 R_{\odot}-1.84$ $R_{\odot}$ when we consider the average fractional radius of the primary component from photometric solution. Same calculation puts $R_{2}$ between $1.45 R_{\odot}-1.52$ $R_{\odot}$. A similar method can be used for the luminosities of the components by using Stefan - Boltzmann law and $T_{\odot}=5770 \mathrm{~K}$. Assuming a lower and upper limits for $T_{1}$ and $T_{2}$ via $\sigma_{T_{1}}=470 \mathrm{~K}$, we can calculate the ranges of $L_{1}$ and $L_{2}$ as $31 L_{\odot}-49 L_{\odot}$ and $5 L_{\odot}-9 L_{\odot}$, respectively.

We show preliminary plots of the components in $\log T_{\text {eff }}-\log L$ plane in Figure 4. Evolutionary tracks for solar abundance $(Z=0.019)$ comes from Girardi et al. (2000). The components of the system seem close to the ZAMS and in good agreement with solar metal abundance. However, spectroscopic analysis is necessary to revise or refine it.

We can make an estimation for the uncertainty of the distance in a sim- 


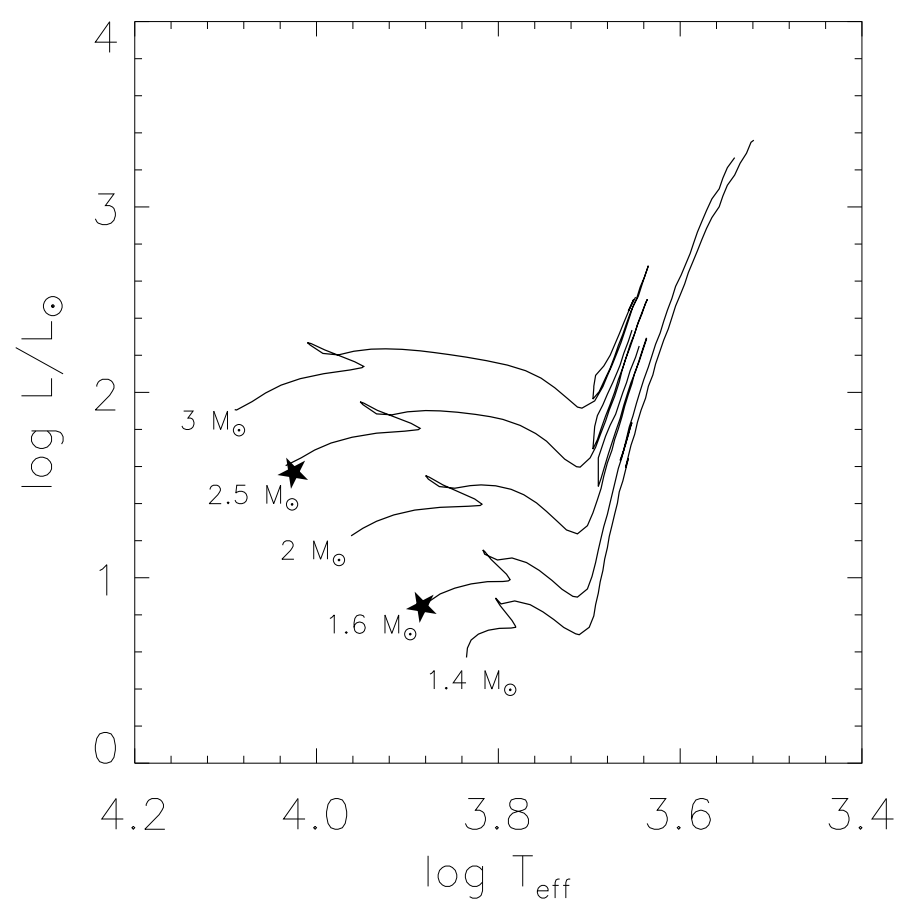

Figure 4: Positions of the components (filled stars) in $\log T_{\text {eff }}-\log L$ plane. All tracks for $Z=0.019$ are from Girardi et al. (2000).

ilar way, as described above. If we assume the same ranges for $q, M_{1}$ and $T_{1}$ as in previous uncertainty estimations, we can calculate the range of absolute bolometric magnitude of the primary component as $1^{m} .01-0^{m} .52$. Here, we adopt bolometric corrections from Gray (2005) for corresponding temperature limits in order to calculate limit visual absolute magnitudes $\left(M_{V}\right)$ of the primary component. Using de-reddened $V$ magnitude and $M_{V}$ limits of the primary component in distance modulus, we can calculate the range of the distance as $329-377 \mathrm{pc}$ and this leads a mean value of $353 \mathrm{pc}$ which is our estimation in previous section. The uncertainty in the distance might be slightly exaggerated since we make a rough uncertainty estimation 
for related parameters. However, our distance estimation differs only by 20 pc from distance value of the cluster (Kharchenko et al., 2005). This confirms the membership of BD +36 3317 to Delta Lyrae cluster. Nevertheless, comprehensive spectroscopic study of the system, in terms of radial velocity measurements, would help to refine or revise the physical properties and distance of the system. Further spectroscopy would also give a chance to check the metal abundance of the system which also contains some hints about the nature of the cluster.

\section{Acknowledgments}

The authors acknowledge generous allotments of observing time at the Ege University Observatory.

\section{References}

Andruk, V., Kharchenko, N., Schilbach, E., Scholz, D., 1995, AN, 316, 225

Anthony-Twarog, B.J., 1984, AJ, 89, 655

Díaz-Cordovés J., Giménez A., 1992, A\&A, 259, 227

Drilling, J. S., Landolt, A. U., 2000, in Cox A. N. (ed.),Allens Astrophysical Quantities, 4th ed., Springer, Berlin, p. 388

Eggen, O.J., 1968, ApJ, 152, 77

Eggen, O.J., 1972, ApJ, 173, 63

Eggen, O.J., 1983, MNRAS, 204, 391 
Girardi, L., Bressan, A., Bertelli, G., Chiosi, C., 2000, A\&AS, 141, 371

Gray, D.F., 2005, The observation and analysis of stellar photospheres, 3rd ed., Cambridge Univ. Press

Kharchenko, N.V., Piskunov, A.E., Röser, S., Schilbach, E., Scholz, R.-D., 2004, AN, 325, 740

Kharchenko, N.V., Piskunov, A.E., Röser, S., Schilbach, E., Scholz, R.-D., 2005, A\&A, 438, 1163

Menzies, J. W., Marang, F, 1996, MNRAS, 282, 313

Sipahi, E., Dal, H.A., Özdarcan, O., 2009, IBVS, 5904, 1

Stephenson, C.B., 1959, PASP, 71, 145

van Hamme, W., 1993, AJ, 106, 2096

Violat-Bordonau, F., Arranz-Heras, T., 2008, IBVS, 5900, 7

Wilson, R.E., Devinney, Edward J., 1971, ApJ, 166, 605

Wilson, R.E., 1979, ApJ, 234, 1054

Wilson, R.E., 1990, ApJ, 356, 613 